\title{
Respiratory System Findings Date Time of Reference Timepoint
}

National Cancer Institute

\section{Source}

National Cancer Institute. Respiratory System Findings Date Time of Reference

Timepoint. NCl Thesaurus. Code C123998.

The date and time of a specific reference point for the respiratory system assessment. 